\title{
Microrheology of colloidal dispersions by Brownian dynamics simulations
}

lleana C. Carpen, and John F. Brady

Citation: Journal of Rheology 49, 1483 (2005); doi: 10.1122/1.2085174

View online: https://doi.org/10.1122/1.2085174

View Table of Contents: http://sor.scitation.org/toc/jor/49/6

Published by the The Society of Rheology

\section{Articles you may be interested in}

A simple paradigm for active and nonlinear microrheology

Physics of Fluids 17, 073101 (2005); 10.1063/1.1960607

Brownian Dynamics simulation of hard-sphere colloidal dispersions

Journal of Rheology 44, 629 (2000); 10.1122/1.551104

Microviscosity, microdiffusivity, and normal stresses in colloidal dispersions

Journal of Rheology 56, 1175 (2012); 10.1122/1.4722880

"Microviscoelasticity" of colloidal dispersions

Journal of Rheology 49, 1449 (2005); 10.1122/1.2085173

Laser tweezer microrheology of a colloidal suspension

Journal of Rheology 50, 77 (2006); 10.1122/1.2139098

Microrheology of colloidal dispersions: Shape matters

Journal of Rheology 52, 165 (2008); 10.1122/1.2821894

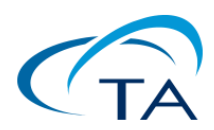

The WORLD'S most VERSATILE platform for RHEOLOGICAL MEASUREMENTS

The Discovery Hybrid Rheometer
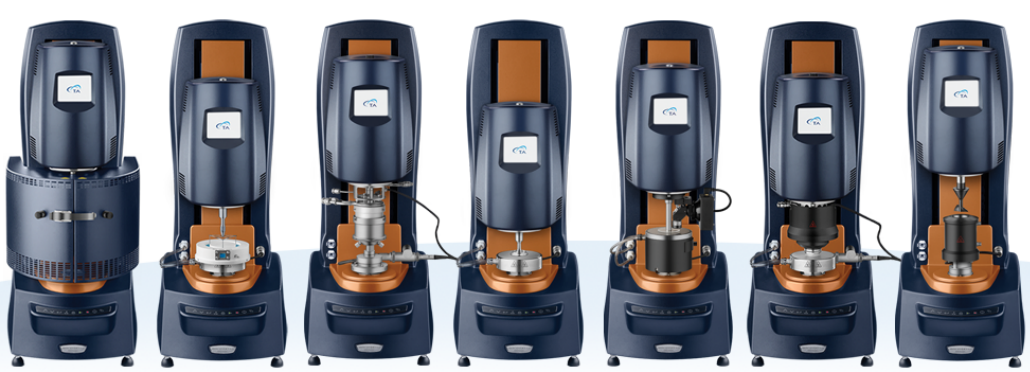


\title{
Microrheology of colloidal dispersions by Brownian dynamics simulations
}

\author{
Ileana C. Carpen \\ Faculty of Science and Technology, University of Twente, P.O. Box 217, \\ 7500 AE Enschede, The Netherlands \\ John F. Brady ${ }^{\text {a) }}$ \\ Division of Chemistry and Chemical Engineering, California Institute of \\ Technology, Pasadena, California 91125 and Faculty of Science and Technology, \\ University of Twente, P.O. Box 217, 7500 AE Enschede, The Netherlands
}

(Received 24 June 2005; final revision received 30 August 2005)

\begin{abstract}
Synopsis
We investigate active particle-tracking microrheology in a colloidal dispersion by Brownian dynamics simulations. A probe particle is dragged through the dispersion with an externally imposed force in order to access the nonlinear viscoelastic response of the medium. The probe's motion is governed by a balance between the external force and the entropic "reactive" force of the dispersion resulting from the microstructural deformation. A "microviscosity" is defined by appealing to the Stokes drag on the probe and serves as a measure of the viscoelastic response. This microviscosity is a function of the Péclet number $(\mathrm{Pe}=F a / k T)$-the ratio of "driven" $(F)$ to diffusive $(k T / a)$ transport-as well as of the volume fraction of the force-free bath particles making up the colloidal dispersion. At low $\mathrm{Pe}$ - in the passive microrheology regime-the microviscosity can be directly related to the long-time self-diffusivity of the probe. As Pe increases, the microviscosity "force-thins" until another Newtonian plateau is reached at large Pe. Microviscosities for all Péclet numbers and volume fractions can be collapsed onto a single curve through a simple volume fraction scaling and equate well to predictions from dilute microrheology theory. The microviscosity is shown to compare well with traditional macrorheology results (theory and simulations). (C) 2005 The Society of Rheology. [DOI: 10.1122/1.2085174]
\end{abstract}

\section{INTRODUCTION}

Rheology, the study of the deformation and flow of matter, has a long history with many of its experimental methods and tools firmly established, and it is in the measurements that one finds the most novelty. However, the end of the last century saw tremendous improvements in imaging technology and computing power, which have enabled scientists to look at rheology on a different level—at the microscopic scale. In the last decade, microrheology - as this type of rheology has come to be called-has gained momentum as a novel tool with novel measurements, useful for studying the properties of

${ }^{a)}$ Author to whom correspondence should be addressed; electronic mail: jfbrady@caltech.edu

(C) 2005 by The Society of Rheology, Inc.

J. Rheol. 49(6), 1483-1502 November/December (2005) 0148-6055/2005/49(6)/1483/20/\$25.00 
materials on a much smaller scale. In this, it is particularly well-suited for examining the properties of soft heterogeneous materials, especially biological materials.

Among the collection of techniques known as microrheology, most involve tracking the motion of a colloidal particle or set of particles in order to infer the properties of the surrounding environment [MacKintosh (1999)]. Due to the small length scales involved, microrheology became practical only with recent advances in technology [although early examples do exist-Freundlich and Seifriz (1922)]; the imaging technology itself also places constraints on the minimum level of error one can expect [Helseth and Fischer (2004)]. Particle-tracking microrheology can be categorized as either passive-tracking the random motion of probe particles due to thermal fluctuations-or active-applying a constant or oscillatory force to the probe particles, for example by using optical tweezers or magnetic fields. Unlike conventional rheology, which studies bulk properties, microrheology allows the measurement of local viscoelastic properties and requires minute material samples, a particular bonus for scarce biomaterials and systems, such as individual cells; for gels, microrheology also avoids the problem of slip at the walls, often encountered with rheometers. Microrheological techniques have been used to study a diverse set of systems: Cells [Guilford et al. (1995); Bausch et al. (1998); Lau et al. (2003)], actin networks [Ziemann et al. (1994); Gisler and Weitz (1999)], gelatin [Freundlich and Seifriz (1922)], deoxyribonucleic acid and polyethylene oxide solutions [Mason et al. (1997)], and the behavior of colloids near the glass transition [Habdas et al. (2004)], as well as fundamental interactions between pairs of colloidal spheres [Crocker (1997)] and entropic forces in binary colloids [Crocker et al. (1999)]. Microrheology has also been proposed as a tool for fundamental physics and for high-throughput material screening [Breedveld and Pine (2003)].

The cornerstone of passive particle-tracking microrheology is the use of the generalized Stokes-Einstein-Sutherland ${ }^{1}$ relation for a sphere in a homogeneous incompressible fluid. For single-particle tracking experiments, this takes the form:

$$
D_{\infty}^{s}=\frac{k T}{6 \pi \eta_{\mathrm{eff}} a},
$$

where $D_{\infty}^{s}$ is the long-time self-diffusivity of the probe, obtained from its mean-squared displacement, $\eta_{\text {eff }}$ is the sought-after effective viscosity of the medium, $k T$ is the thermal energy, and $a$ is the radius of the probe particle. A similar equation applies for two-point microrheology, for which the correlated motion of pairs of particles is used instead of the single-particle mean-squared displacement [Crocker et al. (2000)]. The Stokes-EinsteinSutherland relation translates the microrheological measurement (mean-squared displacement) into the macroscopic measurement (effective viscosity or complex modulus), and is therefore crucial to any type of comparison between micro- and macrorheology. Most applications of microrheology have been in this passive regime and the applicability, or lack thereof, of the generalized Stokes-Einstein-Sutherland equation (1) has been examined [Crocker et al. (2000)]. In the passive regime, the mean-squared displacement of the probe is a measure of the diffusivity, and the macroscopic rheology "equivalent" is the dynamic viscosity; both properties have been extensively studied for colloidal dispersions [e.g., Brady (1994); van der Werff et al. (1989)]. Further, Brady (1994) has shown

${ }^{1}$ It has only recently come to the attention of the scientific and engineering communities that Sutherland ["A dynamical theory of diffusion for nonelectrolytes and the molecular mass of albumin." Philos. Mag. 9, 781-785 (1905)] derived the relationship between the translational diffusion coefficient and the hydrodynamic mobility by the same approach as Einstein, and published the result in the very same year. We propose, therefore, to call this the Stokes-Einstein-Sutherland relation. 
theoretically that the frequency (or time)-dependent self-diffusivity can be compared to the frequency-dependent dynamic viscosity, with good agreement for all frequencies and volume fractions when the different volume fraction scalings $\left[\mathcal{O}(\phi)\right.$ versus $\mathcal{O}\left(\phi^{2}\right)$ in the dilute limit, reflecting the dipolar versus quadrupolar forcing] are taken into account [see also Khair and Brady (in press)].

By contrast, much less work has been done on active microrheology, where the tracked particle is driven by an external force [e.g., the experiments of Habdas and co-workers (2004) and the theoretical work of Brady and co-workers-Squires and Brady (2005); Khair and Brady (in press)]. By its very nature, the passive regime is limited to studying the linear viscoelastic behavior of the material, while the active regime can be used to drive the system out of equilibrium and investigate the material's nonlinear response. In many situations, materials are often subjected to strong flows and large deformations, and therefore it is important to know and understand the nonlinear behavior. Macroscopic rheology takes this into account: Viscosities and other material properties of complex fluids, including colloids [e.g., Bergenholtz et al. (2002)], are measured under varying conditions (shear rates, etc.) to determine the complete behavior. It is the purpose of this work to numerically study active microrheology and the transition from linear to nonlinear behavior. We shall do this using a model complex fluid-a colloidal dispersion - given the availability of complementary results in the colloids literature.

Furthermore, we are interested in how far one may extend the comparison between the micro and macro measurements, given the obvious differences between the forcings employed in these methods. Note that the motion of a probe particle, whether active or passive, does not produce a viscometric flow in the surrounding medium even when the probe is much larger than the characteristic microstructural length scales and is therefore "macroscopic." Thus, the relation between the "microviscosity" inferred from Stokes drag (or the Stokes-Einstein-Sutherland relation in the passive regime) and the macroscopic counterpart is an important and still open question. One we hope to shed some light on by studying a well-defined colloidal system.

In microrheology experiments, the displacement of a probe particle can be induced through the use of magnetic fields and optical tweezers, or alternatively, by having a heavy particle suspended amidst a sea of neutrally buoyant particles. The basic problem reduces to examining the motion of a single particle under the action of an imposed external force and determining how this motion is affected by the response of the material. As the probe moves, it disturbs the local medium, which relaxes through thermally driven motion. Here, we investigate perhaps the simplest viscoelastic fluid-a dispersion of colloidal particles suspended in a Newtonian solvent. The basic problem is illustrated in Fig. 1, and we propose to study active microrheology using Brownian dynamics (BD) simulations, which are particle-level simulations - the dispersion is treated as a collection of spheres in a Newtonian fluid. The particles are monodisperse hard spheres (no overlap or deformation of particles is allowed). Each individual colloidal particle is subject to viscous drag and Brownian motion, but we choose to ignore (for this study) fluidmediated (hydrodynamic) interactions between the particles. The benefits of simulations are two fold: First of all, they provide a means of checking and extending theoretical analyses, and second, they allow us to analyze experimental systems in which different effects can be cleanly separated. Although the removal of hydrodynamic interactions may appear to be a harsh assumption, it can be achieved experimentally with particles subject to a repulsive potential that prevents them from approaching too closely. As Brady and co-workers [Bergenholtz et al. (2002); Khair and Brady (in press)] have noted, even a small difference between the effective and the hydrodynamic radii can strongly decrease the effect of hydrodynamic lubrication interactions. 


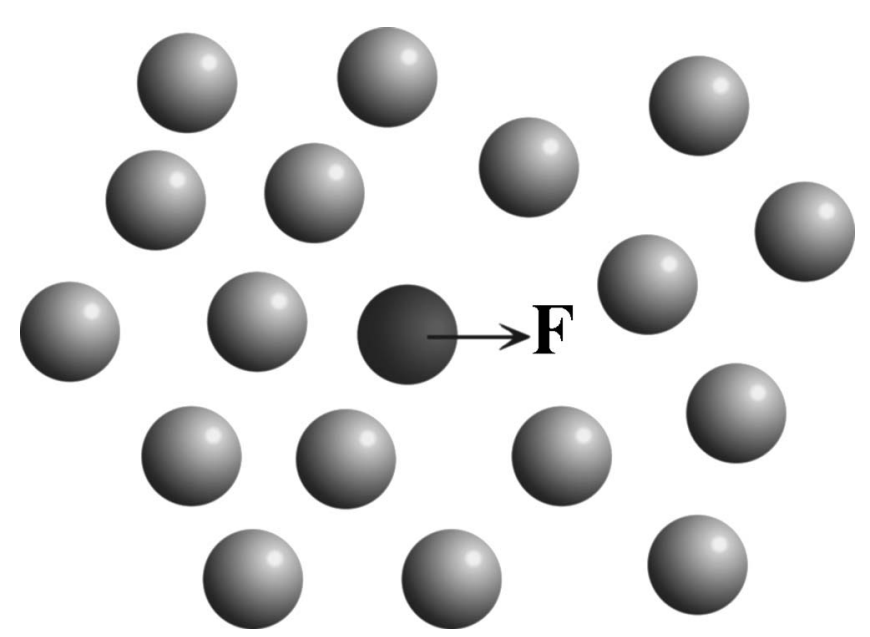

FIG. 1. The model system: a probe particle is dragged by means of an imposed external force through a surrounding suspension of monodisperse force-free bath particles. The relative strength of Brownian ( $U^{B}$ $\left.\sim D / a \sim k T / 6 \pi \eta a^{2}\right)$ to driven motion $\left(U^{F} \sim F / 6 \pi \eta a\right)$ gives the Péclet number $\mathrm{Pe}=F a / k T$ and governs the behavior of the system. Here, $D$ is the Stokes-Einstein-Sutherland diffusivity of a single particle of radius $a$ and thermal energy $k T$ in a fluid of viscosity $\eta$. (Alternatively, the probe particle may be dragged with a constant velocity $U$, in which case $U^{F}=U$ and $\mathrm{Pe}=U a / D$.)

Because the probe's motion is no longer purely Brownian in active microrheology, the probe particle has an average displacement and therefore an average velocity, from which we can define an effective microviscosity of the dispersion through the use of Stokes drag law. This microviscosity is a function of the Péclet number-the ratio of the imposed motion to the thermal motion-and the volume fraction of the freely suspended bath particles. The results of a series of BD simulations are detailed in Sec. IV. The microviscosity exhibits a Newtonian plateau for low Péclet numbers, force thins as the Péclet number is increased, and finally reaches a second plateau region for high Péclet numbers. The microviscosity is also an increasing function of volume fraction, and a simple theory by Squires and Brady (2005) can be used to give appropriate scaling relations that collapse the effective viscosities at different suspension volume fractions and Péclet numbers onto a single universal curve (a brief overview of the dilute theory is given in Sec. III). The dispersion with the highest volume fraction (55\%), displays yield behavior, and the simple scaling arguments of Squires and Brady (2005) only apply at the highest Péclet numbers studied where the motion of the probe "liquefies" the material in its immediate neighborhood. For small Pe, the system is solidlike. We also address the difference in the measurements when the probe is dragged with a constant force versus a constant velocity. These two cases result in different effective viscosities-when the particle is dragged with a constant velocity, the probe cannot move laterally as it passes suspended particles, the suspension is more dissipative and the effective viscosity calculated is higher than for the equivalent constant force measurement.

One of the central issues in the use of microrheology is the relation between the microviscosity and the macroviscosity - the viscosity measured in a conventional macroscopic rheometer-and, therefore, one of the key goals of this study has been to compare our microviscosity results to the results obtained for a homogeneously sheared suspension. A direct comparison can be made to the simulation work of Foss and Brady (2000) who conducted BD simulations of suspensions at some of the same volume fractions as used in this study. In the BD simulations of Foss and Brady (2000), a simple 
shear flow is applied and a shear viscosity (defined in the standard way) obtained, which is dependent on the volume fraction and Péclet number, which for their problem is defined as a ratio of shear to thermal forces. We find that the results for the shear viscosity (both theory and simulations) and the microrheology results agree qualitatively, and almost quantitatively when appropriately interpreted (allowance made for the obvious volume fraction difference in their definitions) - the micro- and macroviscosities both exhibit a force/shear-thinning region and a high Péclet number Newtonian plateau. And, perhaps surprisingly, this good comparison applies even though the probe and bath particles are the same size. We conclude in Sec. V with some final remarks and suggestions for future work.

\section{BROWNIAN DYNAMICS METHOD APPLIED TO MICRORHEOLOGY}

The problem we address is that of a single "probe" particle moving through a surrounding colloidal dispersion with either a constant force or a constant velocity, as illustrated in Fig. 1. While the detailed interparticle interactions and chemistry are often important for specific systems, the most basic property of a complex fluid is a microstructure that can be deformed by an external probe and which returns to equilibrium by thermal motion. The simplest system that captures this basic essence is a colloidal dispersion of neutrally- buoyant monodisperse hard spheres. For microrheology the "probe" particle can be the same as any other colloidal particle (for simplicity the probe and background particles in this study are the same size; in experiments, the sizes are often different), and without any external forcing, its motion is simply diffusive. However, when its motion is driven by an external force, so that it is no longer completely thermal, the probe can drive the surrounding microstructure out of equilibrium, and thereby transitions from passive to active microrheology and from linear to nonlinear behavior.

$\mathrm{BD}$ can be used to simulate colloids at the particle level. The size of colloidal particles is such that there is a clear separation of time and length scales between the particles and the fluid molecules - therefore, the fluid can be treated as a continuum-but the particles are still small enough to be affected by collisions with the fluid molecules and are thus Brownian. The BD method is well-established and has been investigated and expanded by various researchers [a further description of BD and relevant references are given in Allen and Tildesley (1987)]. Our approach is similar to that used by Foss and Brady (2000) for sheared colloidal dispersions.

The motion of the individual particles is governed by the Langevin equation-a balance between the hydrodynamic, Brownian, and any other forces that may be present:

$$
\mathbf{m} \cdot \frac{d \mathbf{U}}{d t}=\mathbf{F}^{H}+\mathbf{F}^{B}+\mathbf{F}^{P} .
$$

Here, $\mathbf{m}$ is the generalized mass/moment of inertia tensor, $\mathbf{U}$ is the particle velocity vector, $\mathbf{F}^{H}$ represents the hydrodynamic forces on the particles; $\mathbf{F}^{B}$ are the Brownian forces; and $\mathbf{F}^{P}$ represents any other deterministic nonhydrodynamic forces that are present, e.g., hard-sphere and external. For colloidal systems, inertia is not important (small Reynolds number) and Eq. (2) reduces to

$$
0=\mathbf{F}^{H}+\mathbf{F}^{B}+\mathbf{F}^{P} .
$$

For BD, there are no hydrodynamic interactions and therefore the forces in Eq. (3) are given simply by

$$
\mathbf{F}^{H}=-6 \pi \eta a \mathbf{U},
$$




$$
\begin{gathered}
\overline{\mathbf{F}^{B}}=\mathbf{0}, \quad \overline{\mathbf{F}^{B}(0) \mathbf{F}^{B}(t)}=2 k T(6 \pi \eta a) \mathbf{I} \delta(t), \quad \text { and } \\
\mathbf{F}^{P}=\mathbf{F}^{H S}+\mathbf{F}^{\mathrm{ext}},
\end{gathered}
$$

where $\eta$ is the solvent viscosity, $a$ is the particle radius, and $k T$ is the thermal energy. The overbars indicate an ensemble average over the thermal fluctuations in the fluid, with the amplitude of the Brownian forcing being given by the fluctuation-dissipation theorem. For microrheology, the external force $\mathbf{F}^{\text {ext }}$ is only applied to the probe particle. For spherical particles in the absence of hydrodynamic interactions, rotation of the particles does not matter.

An integral of Eq. (3) over the time step $\Delta t$ gives the nondimensional displacement equation

$$
\Delta \mathbf{X}=\Delta \mathbf{X}^{B}+\operatorname{Pe}^{\text {ext }} \Delta t+\Delta \mathbf{X}^{H S}
$$

with

$$
\overline{\Delta \mathbf{X}^{B}}=\mathbf{0} \text { and } \overline{\Delta \mathbf{X}^{B} \Delta \mathbf{X}^{B}}=2 \Delta t \mathbf{I} .
$$

Here, time is nondimensionalized by the characteristic diffusive time, $\tau=a^{2} / D$, where $D=k T / 6 \pi \eta a$ is the Stokes-Einstein-Sutherland diffusivity of an isolated particle, length by the particle radius, $a$, the external force by its magnitude, $F$, and the Brownian displacement by its characteristic scale, $\sqrt{k T(6 \pi \eta a)}$. The relative importance of "driven" $\left(U^{F} \sim F / 6 \pi \eta a\right)$ to Brownian $\left(U^{B} \sim D / a \sim k T / 6 \pi \eta a^{2}\right)$ motion is expressed as a Péclet number, $\mathrm{Pe}=U^{F} / U^{B}=F a / k T$. The neglect of the fluid and/or particle inertia requires that the Reynolds number $\operatorname{Re}=\rho U a / \eta \ll 1$. The Reynolds number may also be written as $\operatorname{Re}=\operatorname{Pe}(D / \nu)$, where $\nu$ is the kinematic viscosity of the fluid. For a $1 \mu \mathrm{m}$ particle in water $D / \nu \sim 10^{-6}$ and thus even for very large Péclet number, the Reynolds number is still small and inertia is not important.

At each time step, the positions of the particles are updated first by adding the displacements due to the Brownian and external forces, and second by an iterative method which corrects collisions by applying the hard-sphere force/displacement $\Delta \mathbf{X}^{H S}$. This hard-sphere collision scheme is based on the "potential-free" algorithm of Heyes and Melrose (1993) in which the overlap between pairs of particles is corrected by moving the particles equal amounts along the line of centers, back to contact. This algorithm is "potential free" in that it does not require a specific declaration of a pair potential, although it implements the hard-sphere potential which is infinite if the particles are overlapping and zero otherwise.

The above approach was used to examine the properties of a colloidal dispersion in two cases: With a probe particle acting under either an imposed constant force or constant velocity.

\section{A. Constant force}

For the constant force case, a constant in time (although one could impose an arbitrary time dependence, if desired) external force is exerted on the probe particle. From the displacement of the probe, we can obtain its average velocity resulting from the applied external force, and this may be used to define an effective viscosity for the suspension from Stokes drag. In dimensionless form the probe's mean velocity is given by 


$$
\langle\mathbf{U}\rangle=\frac{\langle\Delta \mathbf{X}\rangle}{\Delta t}=\frac{\left\langle\Delta \mathbf{X}^{B}\right\rangle}{\Delta t}+\operatorname{Pe} \mathbf{F}^{\mathrm{ext}}+\frac{\left\langle\Delta \mathbf{X}^{H S}\right\rangle}{\Delta t},
$$

where the average $\langle\cdot\rangle$ is defined as the average over a time period $M \Delta t$, e.g., $\langle\beta\rangle$ $=(1 / M) \sum_{i=1}^{M} \beta\left(t_{i}\right)$. Noting that the average Brownian displacement is zero, $\left\langle\overline{\Delta \mathbf{X}^{B}}\right\rangle=0$, one obtains

$$
\langle\mathbf{U}\rangle=\operatorname{Pe} \mathbf{F}^{\mathrm{ext}}+\frac{\left\langle\Delta \mathbf{X}^{H S}\right\rangle}{\Delta t}
$$

Returning to dimensional quantities, we define an effective microviscosity for the suspension from Stokes drag:

$$
\frac{\eta_{\mathrm{eff}}}{\eta} \equiv \frac{F_{x}}{6 \pi \eta a\left\langle U_{x}\right\rangle}
$$

where we have taken $x$ to be the direction of the external force. This effective viscosity is a function of the Péclet number and of the volume fraction of bath particles in the dispersion, $\phi=(4 / 3) \pi a^{3} n$, where $n=N / V$ is the number density of bath particles. (In general, this would also be a function of the size ratio of probe to bath particles, which is one here.) In the simulations, the probe is included in the definition of the volume fraction, and therefore there is a $1 / N$ "error" in $\phi$ (the volume fraction of bath particles is slightly lower). However, as the number of particles $N$ used in the BD simulations is 300 or greater, this deviation is not significant.

\section{B. Constant velocity}

Rather than impose a constant force, one can impose the velocity of the probe and measure the force required to maintain the constant velocity. The dynamics are now somewhat different and the simulations must be performed in a slightly different fashion. From the displacement equation (7), we note that in the case of an imposed velocity the probe particle is displaced according to the following (nondimensional) rule [all of the other particles are still displaced according to Eq. (7)]:

$$
\mathbf{X}_{\text {probe }}(t+\Delta t)-\mathbf{X}_{\text {probe }}(t)=\operatorname{Pe} \mathbf{U} \Delta t,
$$

where now the velocity of the probe, $\mathbf{U}$, is known rather than the applied external force, $\mathbf{F}^{\text {ext. }}$. For the probe particle

$$
\Delta \mathbf{X}_{\text {probe }}=\operatorname{Pe} \mathbf{U} \Delta t=\Delta \mathbf{X}^{B}+\operatorname{Pe}^{\mathrm{ext}} \Delta t+\Delta \mathbf{X}^{H S},
$$

from which the external force necessary to maintain the constant velocity can be determined. The Péclet number is now defined based on the magnitude of the imposed velocity, $\mathrm{Pe}=U / U^{B}=U a / D$. In the imposed velocity problem, hard-sphere collisions between particles fall into two categories: Collisions between background particles (no imposed velocity) and collisions between a background particle and the probe particle. The first collision type is the same as before. The second collision type needs to take into account the fact that since the probe is moving with a constant velocity, it is the background particle that needs to be displaced the entire amount back to the contact position and thus the hard-sphere displacements in Eqs. (13) and (14) are minus those of the bath particles that collided with the probe; the probe receives twice the kick it would have gotten in the constant force case for the same overlap.

It is then straightforward to calculate the average external force $\left\langle\mathbf{F}^{\text {ext }}\right\rangle$ exerted on the probe to keep it moving with a constant velocity $\mathbf{U}$ 


$$
\left\langle\mathbf{F}^{\mathrm{ext}}\right\rangle=\mathbf{U}-\frac{\left\langle\Delta \mathbf{X}^{H S}\right\rangle}{\operatorname{Pe} \Delta t},
$$

and from there the effective viscosity is now defined as (dimensional quantities)

$$
\frac{\eta_{\mathrm{eff}}}{\eta} \equiv \frac{\left\langle F_{x}\right\rangle}{6 \pi \eta a U_{x}} .
$$

Note that for constant velocity, the force required fluctuates as the microstructure changes about the moving probe, while at constant force the probe velocity fluctuates in response to the fluctuating environment.

\section{THEORETICAL BACKGROUND}

Theoretical work on active microrheology includes Squires and Brady (2005) (without hydrodynamic interactions between colloidal particles) and Khair and Brady (in press) (including hydrodynamics). We shall focus here on the work of Squires and Brady (2005) because it provides the theoretical analog of our simulations. The authors consider a probe particle being dragged with a constant force (or velocity) in a suspension of other colloidal particles. The particles are subject to a hard-sphere potential and hydrodynamic interactions are neglected. ${ }^{2}$ When the system is in equilibrium, there is a spherically symmetric probability distribution of encountering bath particles, but this symmetry is lost when the probe is induced to move. The motion of the probe is resisted by the viscous drag of the solvent and by the thermal motion of the surrounding particles: As the probe particle moves, it encounters more particles on its front (forming a zone of increased particle density), and has a trailing wake, a zone of depleted particle density, e.g., see Fig. 3. This microstructural deformation creates a resistance to the motion of the probe particle: There is an entropic reactive force proportional to the thermal energy and inversely to the length scale for the deformed microstructure. Near equilibrium-in passive microrheology - the velocity is linear in the applied force and may be directly related to the long-time self-diffusivity

$$
\langle\mathbf{U}\rangle=M_{\infty}^{s} \mathbf{F}^{\mathrm{ext}}=\frac{D_{\infty}^{s}}{k T} \mathbf{F}^{\mathrm{ext}},
$$

where $D_{\infty}^{s}$ and $M_{\infty}^{s}$ are, respectively, the long-time self-diffusivity and mobility [Brady (1994)].

Squires and Brady (2005) also use Stokes drag to define an effective viscosity, which requires the pair-distribution function $g(\mathbf{r})$ - the probability density of finding a bath particle at location $\mathbf{r}$ relative to the probe-in order to solve for the mean probe velocity $\langle\mathbf{U}\rangle$, and the authors assume that the suspension is dilute in order to obtain analytic results. They extend their study to a comparison between the constant force and constant velocity probes, and conclude that the two cases are different (the effective viscosity is higher when dragging the probe with a constant velocity than with a constant force). In addition, they analyze the effect of the size ratio (probe versus bath particles) and com-

\footnotetext{
${ }^{2}$ Note that the inclusion of hydrodynamics does not qualitatively change the behavior of the effective microviscosity in the low Péclet regime, but does qualitatively change its behavior in the high Péclet regime, resulting in "force thickening" of the effective microviscosity in this region [Khair and Brady (in press)]. This is reminiscent of the behavior of sheared suspensions and the transition from shear thinning to shear thickening at high shear rates (Bergenholtz et al. 2002).
} 


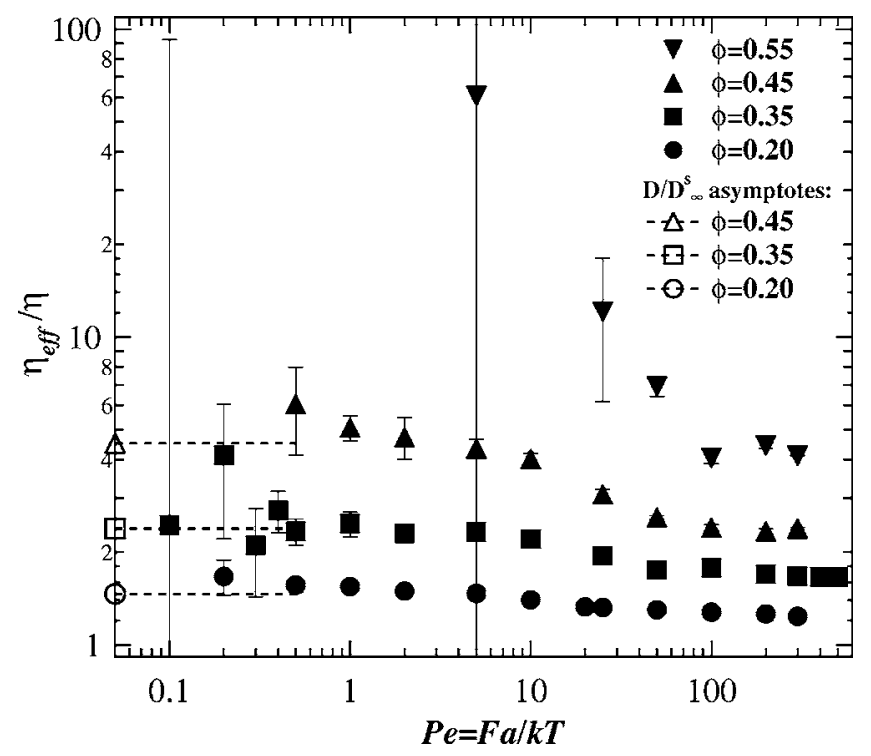

FIG. 2. The effective microviscosity for a constant force probe, $\eta_{\text {eff }} / \eta=F_{x} / 6 \pi \eta a\left\langle U_{x}\right\rangle$, plotted against the Péclet number for different values of the suspension volume fraction. The open symbols/dashed lines represent the $\mathrm{Pe} \rightarrow 0$ asymptotes $\left[\eta_{\text {eff }} / \eta=D / D_{\infty}^{s}(\phi)\right]$ for volume fractions of $20 \%, 35 \%$, and $45 \%$, respectively $\left(D_{\infty}^{s}\right.$ data was obtained from separate pure Brownian $(\mathrm{Pe}=0)$ simulations).

pute fluctuations in the probe motion. In the next section, we compare the (finite volume fraction) simulation results to the results given by their dilute theory and use the theory as a guide for scaling the simulation results.

\section{SIMULATION RESULTS}

In active microrheology, a single-probe particle the same size as the bath particles is pulled through a colloidal dispersion as illustrated in Fig. 1. Although BD simulations are computationally less intensive than Stokesian dynamics and its derivatives, which include hydrodynamic interactions, the BD simulations can still be time consuming. In particular, factors, such as volume fraction and the number of particles in the simulation cell, can lead to a wide variation in computational time. Moreover, because we are interested in measuring the probe's average velocity, and we only have one probe particle per simulation, long and/or multiple runs are required to obtain good accuracy. Recall that for a time average done over a period of $M$ time steps, we have $M$ velocities (for the probe particle) to average, which is a factor of $N$ smaller than if we were allowed to average over all $N$ particles in the simulation cell. To obtain the average velocity, an initial time is subtracted from the run-the time it takes the system to reach steady state-and then a moving average $\left[\langle\beta\rangle=(1 / M) \sum_{i=\zeta}^{M+\zeta-1} \beta\left(t_{i}\right)\right]$ is performed over the rest of the run, with the time period $M \Delta t$ typically equal to 1000 simulation time units. The moving averages are then averaged together to obtain the final average, as well as the deviation from the average. Typical runs are for 5000 simulation time units $\left(\tau=a^{2} / D\right.$ for $\mathrm{Pe} \leqslant 1$ or $\tau$ $=6 \pi \eta a^{2} / F$ for $\left.\mathrm{Pe}>1\right)$ or longer. Many of the simulations were done with 300 particles, with higher Péclet number runs often requiring longer simulation cells (and correspondingly more particles) in order to accommodate the microstructural deformation caused by the probe. Although the particles do not interact hydrodynamically, a probe particle can affect itself due to the periodicity of the simulation cell through a long-ranged deforma- 
tion of the surrounding microstructure: The probe can leave a "trail" that is almost free of particles whose length increases with increasing Péclet number. It is important to be aware of this effect and to size the simulation cell accordingly (see Fig. 3). Further details of the simulations can be found in Carpen (2005).

\section{A. Constant force simulations}

Simulations were done for a range of volume fractions, $0.20 \leqslant \phi \leqslant 0.55$, and Péclet numbers, $0.1 \leqslant P e=F a / k T \leqslant 500$, so as to study the effects of the Péclet number and the volume fraction upon the effective microviscosity measured in active microrheology. Figure 2 shows the effective microviscosity [as given by Eq. (11)] plotted against the Péclet number-the dimensionless force with which the probe is being pulled-for the volume fractions studied: $20 \%, 35 \%, 45 \%$, and $55 \%$. The low Péclet number asymptotes, from Eq. (16), are also shown for $\phi \leqslant 0.45$. The long-time self-diffusivity $\left(D_{\infty}^{s}\right)$ data was independently obtained from separate pure Brownian $(\mathrm{Pe}=0)$ simulations. At small Péclet number, for volume fractions $\phi \leqslant 0.45$, there is a linear relation between the applied force and the velocity resulting in a Newtonian plateau for the microviscosity, and the results approach the passive microrheology limit. The effective microviscosity "force thins" as the Péclet number is increased as the probe deforms the surrounding microstructure. This force thinning in the microrheological context is reminiscent of the shear thinning found in macrorheological measurements of suspensions, and is consistent with the analysis of Squires and Brady (2005). A second Newtonian plateau is achieved at large Péclet numbers.

The effective microviscosity of the dispersion with the highest volume fraction $(\phi$ $=0.55$ ) shows a qualitatively different behavior: This suspension appears to yield, resulting in a diverging viscosity for the lower range of Péclet numbers. The freezing point for a hard-sphere suspension occurs at a volume fraction $\phi \approx 0.494$ and the melting point at $\phi \approx 0.545$. It has been argued that such suspensions also undergo a glass transition at a volume fraction between the freezing point and random close packing $(\phi=0.64)$. Our highest volume fraction $(\phi=0.55)$ suspension is in a solidlike state and therefore displays a very different behavior from that at lower volume fractions, which all corresponded to liquidlike states. However, a probe moved with a strong force (high Pe) is able to "melt" its local environment, and thus the effective viscosity found at high Pe for $\phi=0.55$ starts to behave in a manner similar to that at lower volume fractions.

One can gain a qualitative impression of the suspension behavior through microstructural density profiles relative to the probe (see Figs. 3 and 4). The pictures shown are time averages of the particle density around the probe (center), projected into the $x-y$ plane (where $x$ is the direction of the force/motion of the probe) with the density averaged over the $z$ direction. Close to equilibrium, a probe in a $35 \%$ volume fraction suspension is almost equally likely to find a bath particle in any direction, characteristic of liquids, thus the ring structure seen in the top row of Fig. 3. However, at the highest volume fraction $(\phi=0.55)$ this is no longer the case (Fig. 4, first picture), and the suspension is clearly organized into a solidlike structure.

Figure 3 shows the different density profiles relative to the probe for a volume fraction of 35\% at different Péclet numbers. For small Pe (top row), Brownian motion is strong enough to minimize the effect of the probe, and the density is almost symmetric (as it would be at equilibrium). The break in symmetry about the probe is clearly seen for $\mathrm{Pe}>1$, with the development of a high particle density layer on the front of the probe particle (a sort of cap formed from bath particles, very clear in Fig. 4 for the $\phi=0.55$, $\mathrm{Pe}=100$ case) and a low-density cometlike wake behind the probe. The force thinning of 

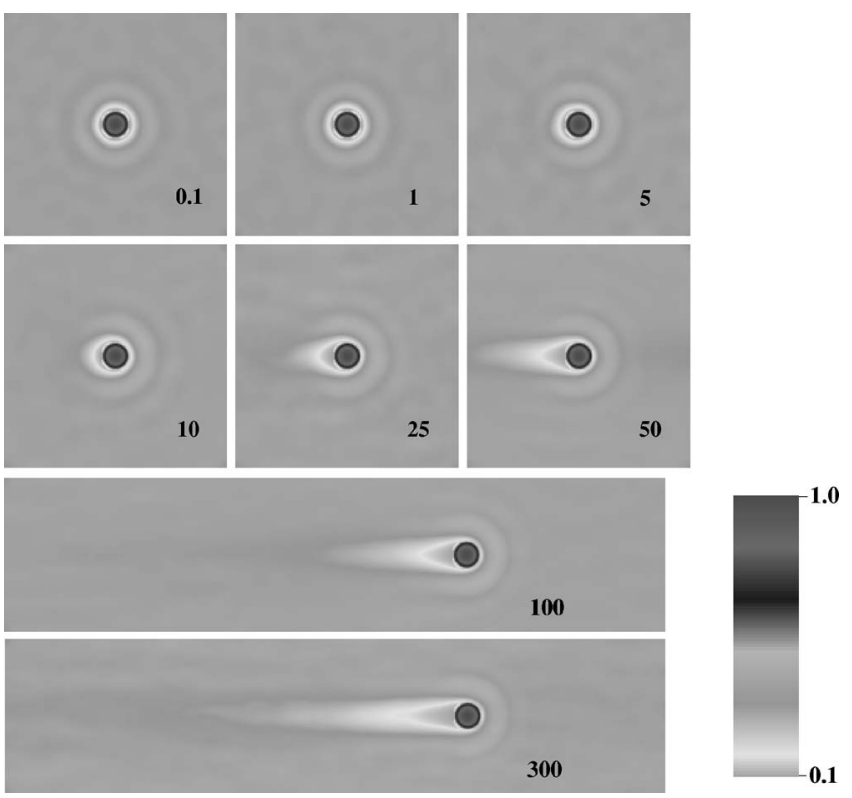

FIG. 3. BD results for the average density (the $x-y$ plane projection) around a probe particle pulled with a constant force for $\phi=0.35$ for different values of the Péclet number. Total number of particles used for the simulations is 300 for the top six (cubic boxes) and 600 for the bottom two (for which the length/width/height ratio is 6/1/1). From left to right: Top row: $\mathrm{Pe}=0.1,1,5$; second row: $\mathrm{Pe}=10,25,50$; third row: $\mathrm{Pe}=100$; bottom row: $\mathrm{Pe}=300$. The images have been processed to improve contrast-in general, lighter areas represent lower density regions and dark high—see grayscale.

the microviscosity is a symptom of this change in the microstructure. The wake grows longer as the Péclet number is increased, reflecting the decreasing ability of thermal/ Brownian motion to heal the microstructure. Squires and Brady (2005) find that in the high Pe limit, the effect of the probe on the microstructure of the dispersion is strongly localized to a thin advection-diffusion boundary layer of thickness $\delta \sim \mathcal{O}(a / \mathrm{Pe})$ on the
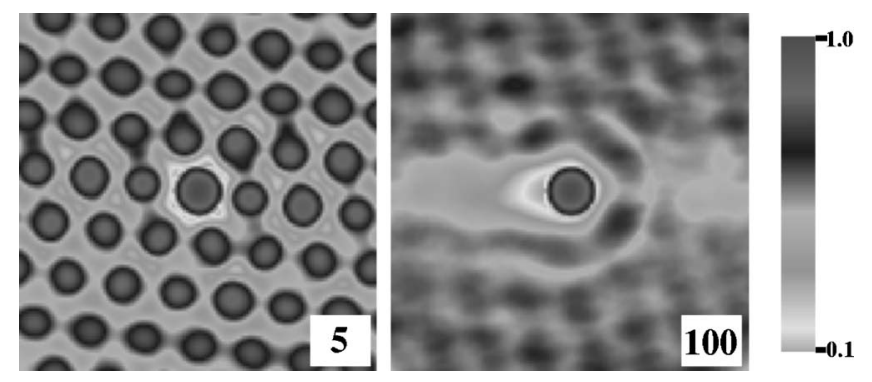

FIG. 4. BD results for the average density (the $x-y$ plane projection) around a probe particle pulled with a constant force for $\phi=0.55$ at $\mathrm{Pe}=5$ and $\mathrm{Pe}=100$. The number of particles used in the simulation cell is 300 . The images have been processed to improve contrast—in general, lighter areas represent lower density regions and dark high—see grayscale.

front of the probe [in which the pair distribution function $g(\mathbf{r})$ is greatly enhanced to a value of $\mathcal{O}(\mathrm{Pe})]$ and a trailing wake devoid of bath particles $(g=0)$. The reactive force of 
the dispersion due to this deformation is $\mathcal{O}(k T / \delta)$, or $\mathcal{O}(k T / a \times \mathrm{Pe})$-as large as the driving force-resulting in a finite value for the microviscosity in the limit of $\mathrm{Pe} \rightarrow \infty$.

For $\phi=0.55$, the system is closely packed, solidlike, and displays yield behaviorforces that are too small cannot break the probe particle out of its local "cage" (see Fig. 4 and note that the figure for $\mathrm{Pe}=5$ corresponds to the time-averaged microstructure relative to the probe-clearly, the probe hops quickly from site to site in the lattice, spending a relatively long time at a lattice site before the next jump). A large force (Péclet number) is required to "melt" the local microstructure and allow the probe to break free. This behavior is in accord with the observations of Habdas and co-workers (2004) in which they studied the behavior of colloids close to the glass transition. This type of yield behavior cannot be predicted by the simple analytical theories used to date (which treat the system as liquidlike), but is captured in the simulations. Note that no effort was made to study the effects of the direction of the external force relative to the suspension lattice on the motion of the probe.

From Fig. 2, we see that the volume fraction can have a very strong effect upon the effective microviscosity: The data spans two orders of magnitude. It is desirable, if possible, to scale out the volume fraction dependence and obtain an universal curve that is independent of $\phi$, as this would eliminate the necessity of making measurements at every volume fraction of interest. In the passive microrheology regime, Brady (1994) showed that the microviscosity is inversely proportional to the long-time self-diffusivity; $D / D_{\infty}^{s}$ are the asymptotic limits at small Pe in Fig. 2. Further, Brady (1994) proposed a simple model for $D_{\infty}^{s}$ :

$$
D_{\infty}^{s} \simeq D_{0}^{s}\left[1+2 \phi g^{\mathrm{eq}}(2 ; \phi)\right]^{-1},
$$

where $D_{0}^{s}$ is the short-time self-diffusivity, which in the absence of hydrodynamic interactions is just the isolated particle Stokes-Einstein-Sutherland diffusivity $D$, and $g^{\mathrm{eq}}(2 ; \phi)$ is the equilibrium value of the pair-distribution function at particle-particle contact, which can be found from the Carnahan-Starling equation of state:

$$
g^{\mathrm{eq}}(2 ; \phi)=\frac{1-\frac{1}{2} \phi}{(1-\phi)^{3}}, \quad \phi \leqslant 0.50 .
$$

At high volume fractions, the long-time self-diffusivity behaves as $D_{\infty}^{s} / D_{0}^{s}$ $\sim\left[\phi g^{\mathrm{eq}}(2 ; \phi)\right]^{-1}$, and as the volume fractions used in our simulations enter this range, we shall use this as our scaling for the microviscosity [although the Carnahan-Starling equation applies for $\phi \leqslant 0.50$, we use the same formula for $\phi=0.55$ - the value obtained is close to the one found for the metastable fluid branch using molecular dynamics by Rintoul and Torquato (1996), 7.96 from Carnahan-Starling versus 8.22 from simulations]. Physically, the term $\phi g^{\mathrm{eq}}(2 ; \phi)$ gives the number of particles contacting the probe.

In addition to scaling the effective viscosity, we must also consider scaling the Péclet number, which was defined based on the characteristic probe speed $U^{F}$ and Brownian speed $U^{B}=D / a$, and may therefore also be affected by the volume fraction. At low Pe, the response time of the microstructure to damage caused by the probe, which is on the scale of the particle radius $a$, scales with the long-time self-diffusivity, $\tau \sim a^{2} / D_{\infty}^{s}(\phi)$. From our previous discussion, the mean velocity of the probe scales as $D_{\infty}^{s}(\phi) F / k T$, and therefore the Péclet number is independent of the volume fraction, i.e., $\mathrm{Pe}=F a / k T$, at least for $\mathrm{Pe}$ up to order unity. For high Péclet number, however, the main contribution to the reactive force, and therefore to the microviscosity, occurs in a thin boundary layer whose length scale is $\delta \sim a / \mathrm{Pe}$. In this case, the response time of the microstructure depends on the time to diffuse this small length, which is governed by the short-time self-diffusivity, $D_{0}^{s}$, 


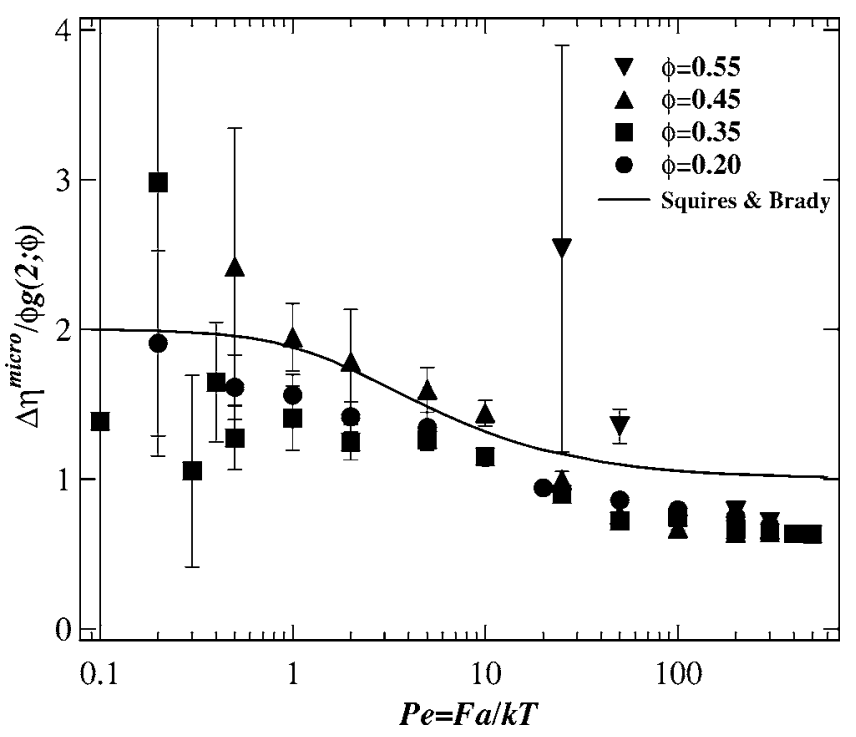

FIG. 5. The solid symbols give the microviscosity increments, $\Delta \eta^{\text {micro }}=\left(\eta_{\text {eff }}-\eta\right) / \eta$, for the constant force system, scaled by $\phi g^{\mathrm{eq}}(2 ; \phi)$, plotted as a function of the Péclet number. This is the same data as in Fig. 2 , rescaled. The solid curve gives the corresponding (dilute) theoretical prediction from Squires and Brady (2005).

rather than the long-time self-diffusivity. The velocity of the probe is given by the product of the mobility and the difference between the external force and the reactive Brownian force (due to the deformation of the microstructure). At high Pe, the reactive Brownian force is proportional to the number of bath particles which collide with the probe $\phi g(2 ; \phi)$ [and $\left.\phi g(2 ; \phi) \sim \operatorname{Pe} \phi g^{\mathrm{eq}}(2 ; \phi)\right]$, and is therefore of the same order of magnitude as the driving force. This results in a mean velocity of the probe that scales as $U^{F}$ $\sim(F / 6 \pi \eta a)\left[1+C \phi g^{\mathrm{eq}}(2 ; \phi)\right]^{-1}$, where $C$ is an $\mathcal{O}(1)$ constant. Therefore, the correct Péclet number in the strong external forcing regime is given by $\mathrm{Pe}=(F a / k T) /[1$ $\left.+C \phi g^{\mathrm{eq}}(2 ; \phi)\right]$, i.e., the rescaled Péclet number is a function of the volume fraction. The factor $\phi g^{\mathrm{eq}}(2 ; \phi)$ varies from 0.35 to 2.09 for most of the range of volume fractions considered in this study ( 4.5 for the $55 \%$ system); the combination $\left[1+C \phi g^{\mathrm{eq}}(2 ; \phi)\right]$ thus may give a weak to moderate dependence on volume fraction. Furthermore, this will just cause a lateral shift in the data at high Pe where they are already in the high-Pe Newtonian plateau and therefore would be hard to discern. Thus, we shall not scale the Péclet number with $\phi$ at high Pe. The scaling of the Péclet number and the microviscosity is discussed in more detail by Squires and Brady (2005).

Although the effective microviscosity of the suspension contains a contribution from the solvent, the relevant contribution is that caused by the microstructural deformation. Therefore, we define the microviscosity increment $\Delta \eta^{\text {micro }}$ as

$$
\Delta \eta^{\text {micro }}=\frac{\eta_{\text {eff }}}{\eta}-1
$$

in order to focus on the effect of the microstructure. In Fig. 5, we plot the microviscosity increment scaled by $\phi g^{\mathrm{eq}}(2 ; \phi)$, as a function of the (unscaled) Péclet number. This scaling provides a significant collapse of the data-note that the scaled viscosity is now on a linear, rather than logarithmic, scale-which shows the features observed before: low and high Péclet number Newtonian plateaus and force thinning at intermediate values 


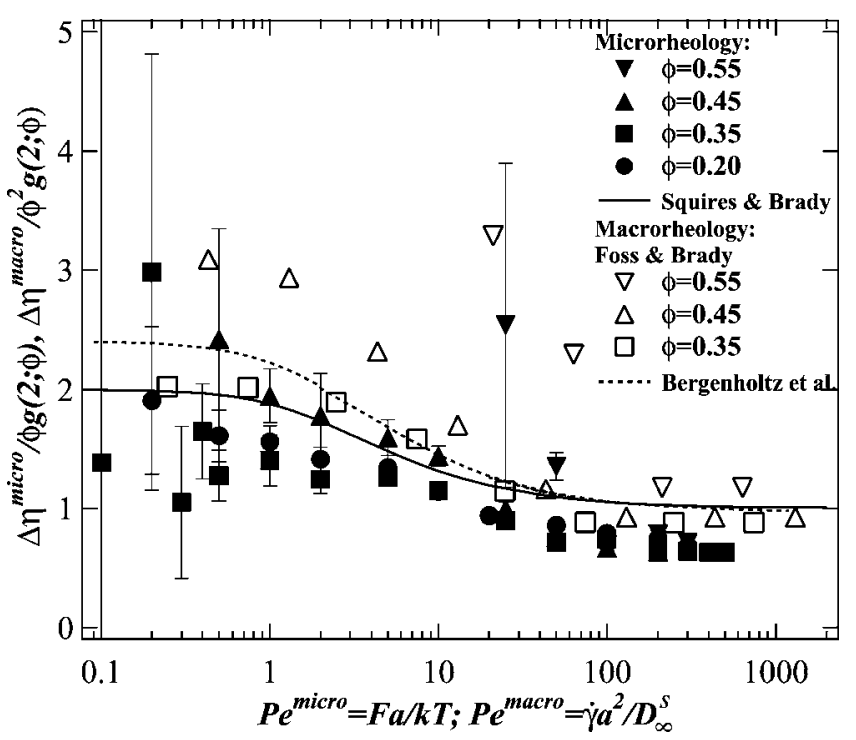

FIG. 6. The solid symbols give the microviscosity increment $\Delta \eta^{\text {micro }}=\left(\eta_{\text {eff }}-\eta\right) / \eta$ for the constant force system, scaled by $\phi g^{\mathrm{eq}}(2 ; \phi)$, plotted as a function of the Péclet number. Shown also are the dilute theory results of Squires and Brady (2005) for the microviscosity (solid line), and the dilute theory results of Bergenholtz and co-workers (2002) (dashed line) and BD simulation results of Foss and Brady (2000) (open symbols) for the macroviscosity.

of the Péclet number. In addition, we can see that even the high volume fraction data $(\phi=0.55)$, joins the universal curve at high Péclet number. At this point, the force on the probe becomes strong enough to locally melt the microstructure and thus the suspension behaves (locally) as a liquid. Plotted also is the dilute theory prediction of Squires and Brady (2005) (solid line). Qualitative and quantitative agreement between the scaled microviscosity increments is excellent for all volume fractions (other than $\phi=0.55$ ).

One of the main goals of this study is to compare micro- and macrorheological measurements. Figure 6 shows the scaled microviscosity simulation data together with the dilute microrheology theory prediction (solid line) of Squires and Brady (2005), compared to data from both dilute theory [Bergenholtz et al. (2002)] (dashed line) and BD simulation results at matching volume fractions [Foss and Brady (2000)] (open symbols) for the macroviscosity. The shear viscosity increment, $\Delta \eta^{\text {macro }}=\eta^{\text {macro }} / \eta-1-2.5 \phi$ (both the solvent and Einstein's single-particle viscosity correction are removed), is plotted against a Péclet number based on the shear rate, $\mathrm{Pe}=\dot{\gamma} a^{2} / D_{\infty}^{s}$, where $\dot{\gamma}$ is the shear rate, and the long-time self-diffusivity is the appropriate diffusivity for the microstructural relaxation. ${ }^{3}$ In addition, the microviscosity increment is $\mathcal{O}(\phi)$, while the macroviscosity increment is $\mathcal{O}\left(\phi^{2}\right)$, and this has been taken into account when scaling the data. Qualitative (and perhaps even quantitative) agreement between the micro and macroviscosity increments is evident. At low Péclet numbers (low shear, low force) both display a

${ }^{3}$ In macrorheology, as in microrheology, at high Péclet number there is a boundary layer at particle-particle, contact that is responsible for the high Pe Newtonian plateau in the viscosity. The relaxation time for the boundary layer, and hence the stress, is governed by the short-time self-diffusivity, which in the absence of hydrodynamic interactions is just the isolated particle value. Thus, the Péclet number should not be scaled with $\phi$ at high Pe as was done in Fig. 5. Not scaling the Péclet number, however, would cause an indiscernible lateral shift in the data at high Pe. 


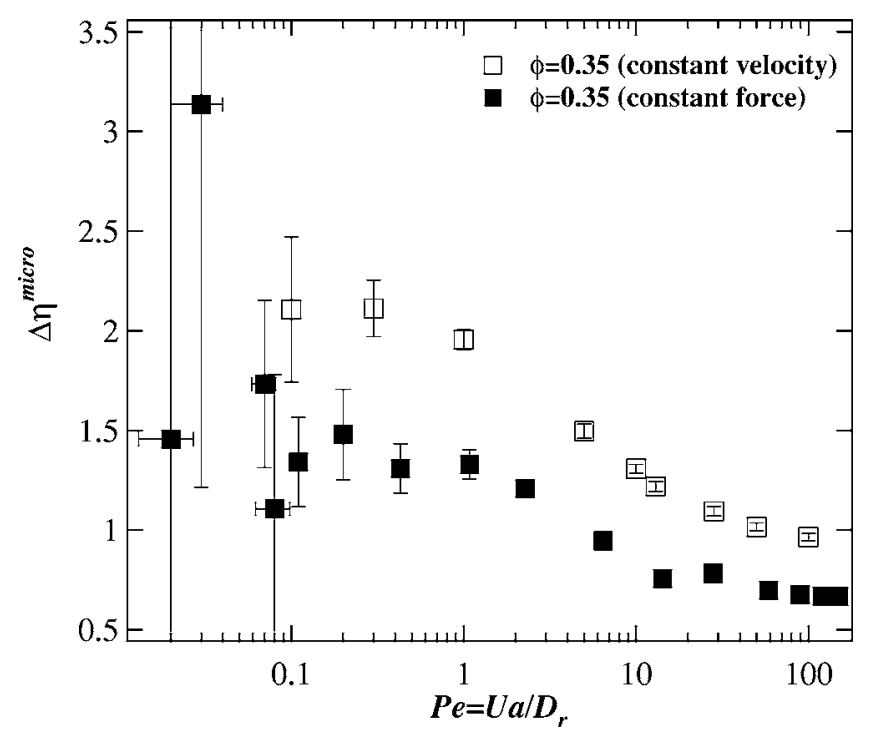

FIG. 7. The viscosity increment of the constant velocity system (effective viscosity minus the solvent contribution, where $\eta^{\text {eff }} / \eta=\left\langle F_{x}\right\rangle / 6 \pi \eta a U_{x}$ ), plotted against the velocity-based Péclet number $\mathrm{Pe}=U a / D_{r}$ for a suspension volume fraction of $35 \%$. Also shown is the equivalent constant force data.

Newtonian plateau; as the Péclet number is increased, the viscosity decreases-force thinning in the microrheology case and shear thinning in the macrorheology case-until a second Newtonian plateau is reached at high Péclet numbers. While this nearly quantitative agreement only applies to the viscosity increments, these reflect the underlying microstructural dynamics responsible for force or stress. Therefore, even though the micro- and macroviscosities are fundamentally different and correspond to distinct forcings, the viscosity increments evidently reflect the same microstructural physics and are in remarkable agreement for all Péclet numbers.

\section{B. Constant velocity simulations}

The comparison in Fig. 6 is not proper in the sense that the microrheology data are measured at fixed force, while the macrorheology data is at fixed shear rate. The correct comparison should be done between the fixed velocity and the fixed shear rate systems, or between fixed force and fixed stress. In macrorheology at the pair-level, the viscosity measured at fixed stress is the same as at fixed shear rate, and it is therefore acceptable to compare to the dilute macrorheology theoretical prediction. However, even at the pair level the two microrheology systems are different. Squires and Brady (2005) have shown that the effective microviscosity in the constant velocity case is larger than in the constant force case (they predict a factor of 2 difference for equal-sized probes and bath particles), essentially since at fixed velocity the probe must push its way past all the bath particles, while at fixed force it can move sideways if necessary to get past a bath particle, and thereby experience less resistance. Almog and Brenner (1997) also found a difference for falling-ball rheometry $(\mathrm{Pe} \rightarrow \infty)$.

In Fig. 7, the microviscosity increment obtained in the constant velocity case is plotted along with the constant force results for a volume fraction of 35\%. As mentioned in Sec. II B, the Péclet number for the constant velocity case is defined based on the imposed velocity rather than the force, $\mathrm{Pe}=U a / D_{r}$. We use the relative diffusivity between the 
bath and the probe, $D_{r}$, which is simply the Stokes-Einstein-Sutherland diffusivity for an isolated particle in the constant velocity case (only the bath particles diffuse), and twice that in the constant force case. The constant force data is also plotted as a function of a velocity-based Péclet number, using the calculated average velocity of the probe. The qualitative behavior is the same in both cases. The main difference is quantitative-the constant velocity system is more dissipative as the probe has to force every bath particle it encounters away, which requires a stronger push than the equivalent constant force probe. The ratio of viscosity increments is approximately $3 / 2$ over the entire range of Péclet numbers, slightly lower than the factor of 2 predicted by the dilute theory.

As a final note, it is clear in microrheology that constant force and constant velocity probing are fundamentally (and observably) different. This then raises the question in macrorheology as to whether constant shear rate and constant stress experiments are also different. It has generally been assumed that the viscosities determined from the two situations are the same (for homogeneous deformations with no shear banding), and indeed this can be shown to be true in the linear-response regime (small $\mathrm{Pe}$ ). In the nonlinear regime, however, it has not been proven that constant stress and constant shear rate experiments give the same rheological behavior. The expectation that they do comes from the fact that the shear rate fluctuations that exist in a constant stress experiment should decrease with the system size and thus vanish in the thermodynamic limit. If the fluctuations do not vanish, say because the system is near a critical point (near a yield stress, for example), then the two situations may be different and their assumed equivalence should be re-examined.

\section{Fluctuations: Constant force microrheology}

One of the advantages of microrheological techniques is that they allow the study of fluctuations in addition to averages. When the probe particle is much larger than the typical length scale of the surrounding medium, as would be the case for a macrorheology experiment, the velocity fluctuations induced by the surrounding suspension may not be observable. However, if the probe size is comparable to the medium length scale, such fluctuations are important and can be considered as a type of noncontinuum effect. For some materials (e.g., living cells and other small or highly heterogeneous systems), the fluctuations in material properties may play a more significant role than the averages. Although we have not varied the size of our probe for this study, we can examine the behavior of velocity fluctuations as a function of Péclet number and volume fraction. For a constant external force the particle velocity will fluctuate, both in the direction of mean motion and in the transverse directions.

In Figs. 8 and 9, fluctuation results are shown for all volume fractions studied. Here, $U_{\|}$is the fluctuation in the velocity component parallel $(x)$ to the forcing direction and $U_{\perp}$ is the fluctuation in the velocity component transverse $(y$ or $z$ ) to the forcing direction. The dimensionless sampling time step is 0.001 . The mean-squared velocity fluctuations (nondimensional) are shown in Fig. 8. In the case of the transverse fluctuations, there is an additional average over the two (identical) transverse directions, i.e., $\left\langle U_{\perp} U_{\perp}\right\rangle$ $=\left(\left\langle U_{y} U_{y}\right\rangle+\left\langle U_{z} U_{z}\right\rangle\right) / 2$. The mean-squared velocity fluctuations decrease with increasing forcing/Péclet number. There does not appear to be any significant difference between the parallel and transverse directions at low Péclet number, but the results at high Pe show some difference, which are clear when we examine the ratio $\left\langle U_{\|} U_{\|}\right\rangle /\left\langle U_{\perp} U_{\perp}\right\rangle$ shown in Fig. 9. The dashed lines in this figure are the asymptotic limits given by the simple dilute theory of Squires and Brady (2005). The increasing anisotropy with increasing Péclet number between the fluctuations oriented parallel versus transverse to the imposed force 


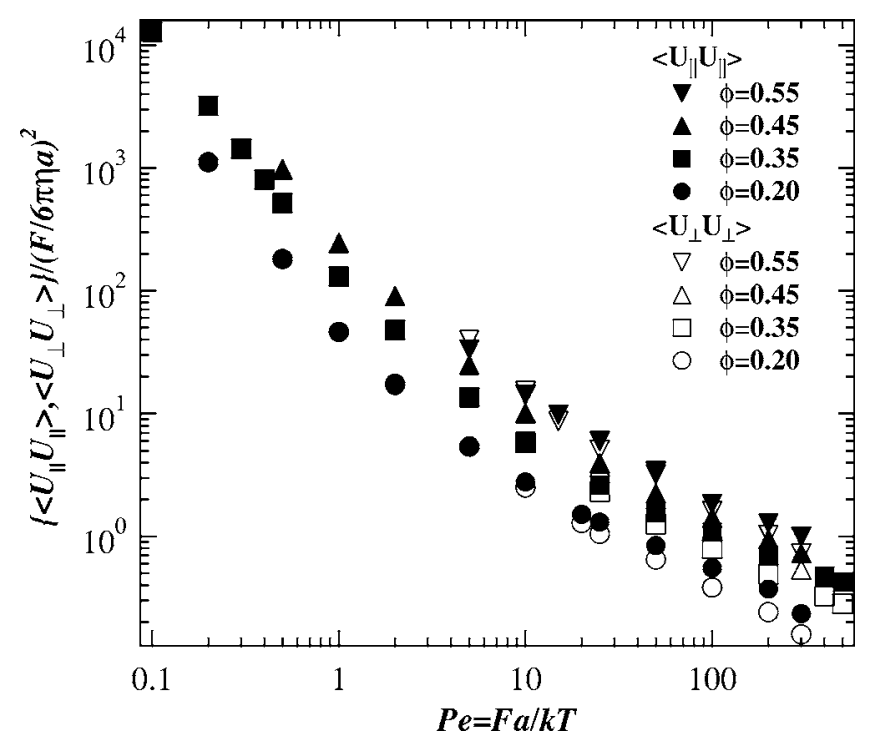

FIG. 8. The averaged velocity fluctuations for the constant force system plotted against $\mathrm{Pe}=F a / k T$ : $U_{\|}$is the fluctuation in the velocity component parallel to the forcing direction; $U_{\perp}$ is the fluctuation in the velocity component transverse to the forcing direction.

is clear. At high Pe, there is an increased probability of collisions on the "front" of the probe, and although these will partially contribute to transverse fluctuations (unless the collision is perfectly "head on"), they will mainly contribute to parallel fluctuationsleading to the pronounced anisotropy.

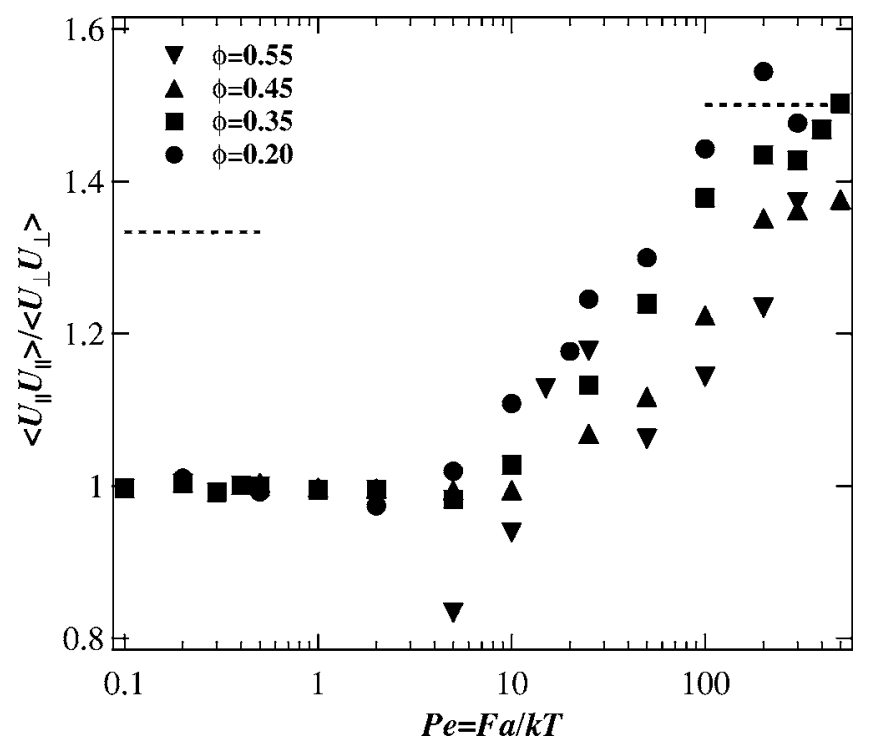

FIG. 9. The averaged velocity fluctuations for the constant force system plotted against $\mathrm{Pe}=F a / k T$. $U_{\|}$is the fluctuation in the velocity component parallel to the forcing direction; $U_{\perp}$ is the fluctuation in the velocity component transverse to the forcing direction. The dashed lines are the low and high Pe asymptotic predictions given by the simple dilute theory. 
The simulation results approach the dilute theory limit for high Péclet numbers, but it is unclear whether the ratio will continue growing or will asymptote to the theory prediction. The relative fluctuations are smaller for low Pe than for high Pe, as the dilute theory suggests, but are lower than the $\mathrm{Pe} \ll 1$ theoretical limit. The agreement at high $\mathrm{Pe}$ may be due to fluctuations in the Pe $\gg 1$ limit caused mostly by collisions with particles in the high particle density shell on the front of the probe-the boundary layer-with a clear directionality (on the average) regardless of the overall concentration, whereas in the small Pe limit the directionality of the collisions may be affected by the relatively large concentrations present.

\section{CONCLUSIONS}

Microrheology presents great opportunities in the study of complex fluids-from biomaterials to colloids to gels-but it also presents challenges in terms of reconciling its results with those of traditional macrorheology. This has, in many respects, limited the use of microrheology to the fairly well understood "passive" regime of unforced probes, which only measures the linear viscoelastic properties of the material. However, the recent theoretical analyses by Squires and Brady (2005) and Khair and Brady (in press) have extended our understanding of microrheology in colloidal dispersions to the nonlinear "active" regime. The work shown here complements these theoretical analyses, presenting a study of active microrheology in colloids of finite and high volume fractions and providing a check on the theoretical predictions. The colloidal system chosen was well defined and well studied, allowing for a comparison to macrorheological results.

The agreement between the simple theory [Squires and Brady (2005)] and the simulation results was very good for the effective viscosity data, although there was no clear agreement and some differences between the relative fluctuations measured at finite volume fractions versus the dilute regime. The simulations also agreed with the theoretical analysis in showing that the viscosity measured when the probe is dragged with a constant velocity is consistently higher than in the constant force case, symptomatic of the greater resistance encountered when the probe cannot deviate from its path and has to force all bath particles out of its way. The simple theory also provided an excellent guide in terms of scaling, allowing us to collapse the effective microviscosity increment data for different volume fractions onto a single universal curve (with the exception of the high volume fraction, $55 \%$, case, for $\mathrm{Pe}<100$ ). The apparent yield behavior shown by the $55 \%$ dispersion was also seen in experiments at high volume fractions [Habdas et al. (2004)]; it is not an effect that can be predicted by the simple dilute theory.

When interpreted as an effective viscosity, the microrheological measurements show force thinning that closely resembles the shear thinning of the macroviscosity for sheared suspensions. One of the most significant conclusions of this study is that the effective viscosity measured by microrheology shows the same qualitative (and almost quantitative) behavior as the viscosity measured using traditional rheology. However, one must take into consideration the different volume fraction scalings (in particular, the relevant dilute theories show that the microviscosity scales with $\phi$, while the macroviscosity scales with $\phi^{2}$ ). Yet, with the correct scaling, the quantitative differences are small, despite the fact that the two are fundamentally different-dipole versus quadrupole forcing, nonviscometric versus viscometric flow, etc. Evidently, the essence of microstructural deformation and relaxation is captured by both micro and macro measurements. The microrheological studies also show a clear distinction between constant force and constant velocity probes, raising the question as to whether this distinction-constant shear rate versus constant stress-is also manifest in nonlinear macrorheology. 
The current study ignored the effect of hydrodynamic interactions. However, we know from the behavior in macrorheological experiments and the theoretical work of Khair and Brady (in press) that such interactions play an important role at higher Péclet numbers, where they result in force (or shear) thickening. It would be interesting to perform the analogous microrheological simulations with hydrodynamics included and compare with their theoretical predictions as well as to macrorheology results. One can also consider the effects of factors, such as the probe size (relative to the bath particles), size polydispersity in general, and interactions between multiple probes-these and other parameters may be very important in the microrheology context. Microrheology measurements are more strongly affected by the character of the material and the particulars of the probing method than the equivalent macrorheology measurements: On the positive side, this indicates that microrheological techniques can be quite sensitive. However, one should keep in mind, when comparing between micro and macro, that current microrheology measurements and techniques focus on obtaining the microviscosity, a scalar quantity, and cannot provide the full stress tensor for the material (at least with a single-spherical particle). At the very least, microrheology can be used to provide information complementary to that obtained by macrorheology techniques and merits further study and development.

\section{ACKNOWLEDGMENTS}

The authors would like to thank Todd Squires and Aditya Khair for valuable discussions.

\section{References}

Allen, M. P., and D. J. Tildesley, Computer Simulation of Liquids (Oxford University Press, Oxford, UK, 1987).

Almog, Y., and H. Brenner, "Non-continuum anomalies in the apparent viscosity experienced by a test sphere moving through an otherwise quiescent suspension," Phys. Fluids 9, 16-22 (1997).

Bausch, A. R., F. Ziemann, A. A. Boulbitch, K. Jacobson, and E. Sackmann, "Local measurements of viscoelastic parameters of adherent cell membranes by magnetic bead microrheometry,” Biophys. J. 75, 2038-2049 (1998).

Bergenholtz, J., J. F. Brady, and M. Vicic, “The non-Newtonian rheology of dilute colloidal suspensions," J. Fluid Mech. 456, 239-275 (2002).

Brady, J. F., "The long-time self-diffusivity in concentrated colloidal dispersions," J. Fluid Mech. 272, 109-133 (1994).

Breedveld, V., and D. J. Pine, "Microrheology as a tool for high-throughput screening," J. Mater. Sci. 38, 4461-4470 (2003).

Carpen, I. C., "Studies of suspension behavior. I. Instabilities of non-Brownian suspensions. II. Microrheology of colloidal suspensions," PhD thesis, California Institute of Technology 2005).

Crocker, J. C., "Measurement of the hydrodynamic corrections to the Brownian motion of two colloidal spheres," J. Chem. Phys. 106, 2837-2840 (1997).

Crocker, J. C., M. T. Valentine, E. R. Weeks, T. Gisler, P. D. Kaplan, A. G. Yodh, and D. A. Weitz, "Two-point microrheology of inhomogeneous soft materials," Phys. Rev. Lett. 85(4), 888-891 (2000).

Crocker, J. C., J. A. Matteo, A. D. Dinsmore, and A. G. Yodh, "Entropic attraction and repulsion in binary colloids probed with a line optical tweezer," Phys. Rev. Lett. 82, 4352-4355 (1999).

Foss, D. R., and J. F. Brady, "Brownian Dynamics simulation of hard-sphere colloidal dispersions," J. Rheol. 44(3), 629-651 (2000).

Freundlich, H., and W. Seifriz, "Über die elastizität von sollen und gelen," Z. Phys. Chem., Stoechiom. Verwandtschaftsl. 104, 233-261 (1922). 
Gisler, T., and D. A. Weitz, "Scaling of the microrheology of semidilute F-actin solutions," Phys. Rev. Lett. 82, 1606-1609 (1999).

Guilford, W. H., R. C. Lantz, and R. W. Gore, "Locomotive forces produced by single leukocytes in-vivo and in-vitro," Am. J. Physiol. 37, C1308-C1312 (1995).

Habdas, P., D. Schaar, A. C. Levitt, and E. R. Weeks, "Forced motion of a probe particle near the colloidal glass transition," Europhys. Lett. 67(3), 477-483 (2004).

Helseth, L. E., and T. M. Fischer, "Fundamental limits of optical microrheology," J. Colloid Interface Sci. 275 , 322-327 (2004).

Heyes, D. M., and J. R. Melrose, "Brownian dynamics simulations of model hard-sphere suspensions," J. Non-Newtonian Fluid Mech. 46, 1-28 (1993).

Khair, A. S., and J. F. Brady, "Single particle motion in colloidal dispersions," J. Fluid Mech. (in press).

Lau, A. W. C., B. D. Hoffman, A. Davies, J. C. Crocker, and T. C. Lubensky, "Microrheology, stress fluctuations, and active behavior of living cells," Phys. Rev. Lett. 91, 198101 (2003).

MacKintosh, F. C., and C. F. Schmidt, "Microrheology," Curr. Opin. Colloid Interface Sci. 4, 300-307 (1999).

Mason, T. G., K. Ganesan, J. H. vanZanten, D. Wirtz, and S. C. Kuo, "Particle tracking microrheology of complex fluids,” Phys. Rev. Lett. 79, 3282-3285 (1997).

Rintoul, M. D., and S. Torquato, “Computer simulations of dense hard-sphere systems,” J. Chem. Phys. 105, 9258-9265 (1996).

Squires, T. M., and J. F. Brady, “A simple paradigm for active and nonlinear microrheology," Phys. Fluids 17(7) 073101 (2005).

Van der Werff, J. C., C. G. de Kruif, C. Blom, and J. Mellema, "Linear viscoelastic behavior of dense hardsphere dispersions," Phys. Rev. A 39(2), 795-807 (1989).

Ziemann, F., J. Radler, and E. Sackmann, "Local measurements of viscoelastic moduli of entangled actin networks using an oscillating magnetic bead micro-rheometer," Biophys. J. 66, 2210-2216 (1994). 